\title{
АЛГОРИТМ СОКРАЩЕННОГО ВЫЧИСЛЕНИЯ ОБРАТНОГО ДИСКРЕТНОГО ПРЕОБРАЗОВАНИЯ ФУРЬЕ
}

\author{
(Представил Н. Алумяэ)
}

\section{1. Введение}

Прямое преобразование Фурье независимо от вида входной переменной (вещественная или комплексная) приводит к комплексному выходному результату, причем при вещественном входном массиве достаточным является вычисление нижней или верхней половины спектра.

Поскольку дискретное преобразование вещественной переменной можно вычислить с помощью значительно меньшего количества математических операций $\left[{ }^{1}\right]$, то представляет интерес осуществить по косинусному и синусному преобразованиям обратное преобразование, не прибегая к комплексному представлению спектра.

Для обратного дискретного преобразования требуется выполнить столько же математических операций, сколько их в алгоритме сокращенного вычисления [ $\left.{ }^{1}\right]$.

\section{2. Постановка задачи и ее предварительный анализ}

Имеются

$$
X C(K)=\sum_{I=0}^{N-1} Y(I) \cos (2 \pi I K / N), \quad K=\overline{0, N / 2},
$$

и

$$
\begin{aligned}
& X S(K)=\sum_{I=1}^{N-1} Y(I) \sin (2 \pi I K / N), \quad K=\overline{1,(N / 2-1)}, \\
& N=2^{* *} L P .
\end{aligned}
$$

По $X C(K)$ и $X S(K)$ требуется восстановить $Y(I)$ (вещественные).

Рассмотрим отдельно восстановление по четным и нечетным гармоникам, т. е. учтем, что

$$
\begin{aligned}
& X C(2 K)=\sum_{I=0}^{N / 2-1} Y 2(I) \cos (2 \pi I K /(N / 2)), \\
& X S(2 K)=\sum_{I=1}^{N / 2-1} Y 2(I) \sin (2 \pi I K /(N / 2)), \\
& X C(2 K+1)=\sum_{I=0}^{N / 2-1} Y(1)(I) \cos (2 \pi I(2 K+1) / N),
\end{aligned}
$$




$$
\begin{array}{ll}
X S(2 K+1)=\sum_{I=1}^{N / 2-1} Y 1(I) \sin (2 \pi I(2 K+1) / N), \\
Y 2(I)=Y(I)+Y(I+N / 2), \quad I=\overline{0,(N / 2-1)} \\
Y 1(I)=Y(I)-Y(I+N / 2), \quad I=\overline{0,(N / 2-1)}
\end{array}
$$

Если по (3)-(6) определяются $Y 2(I)$ и $Y 1(I)$, тогда

$$
\begin{aligned}
& Y(I)=(Y 2(I)+Y 1(I)) / 2, \quad I=\overline{0,(N / 2-1)} \\
& Y(I+N / 2)=(Y 2(I)-Y 1(I)) / 2, \quad I=\overline{0,(N / 2-1)} .
\end{aligned}
$$

и

Кроме того, разбиением $K$ на четные и нечетные в формулах (3) и (4) осуществляется дальнейшее разбиение $Y 2(I)$ на подгруппы, каждая из которых восстанавливается таким же образом как $Y 1(I)$.

Формулы (5) и (6) определяют нечетное трансформантное преобразование (1). Таким образом, задача сведена к обратным нечетным трансформантным преобразованиям (ОНТП) и сумма-разностным преобразованиям типа (9), (10).

\section{3. Обратное нечетное трансформантное преобразование}

Нечетное трансформантное преобразование представляется формулами $\left[{ }^{1}\right]$ :

$X C(2 K+1)=C 1_{N}(2 K+1)+C 1_{N}{ }_{2}(2 K+1)+\ldots+C 1_{8}(2 K+1) \pm Y 1(0)$,

$X S(2 K+1)=S 1_{N}(2 K+1)+S 1_{N / 2}(2 K+1)+\ldots+S 1_{8}(2 K+1) \pm Y 1(N / 4)$,

$C 1_{N / R}(2 K+1)=\sum_{I=0}^{N / 4 R-1} Y 1(R(2 I+1)) \cos (2 \pi(2 I+1)(2 K+1) /(N / R))$,

$S 1_{N / R}(2 K+1)=\sum_{I=0}^{N / 4 R-1} Y 1(R(2 I+1)) \sin (2 \pi(2 I+1)(2 K+1) /(N / R))$,

$$
R=2^{r}, \quad r=\overline{0,(L P-3)}, \quad K=\overline{0,(N / 4 R-1)} .
$$

Учитывая, что

$$
\begin{gathered}
X C(2 K+1)=C 1_{N}(2 K+1)+X C_{N / 2}(2 K+1), \\
X S(2 K+1)=S 1_{N}(2 K+1)+X S_{N / 2}(2 K+1), \\
C 1_{N / R}(N / 4 R-(2 K+1))=-C 1_{N / R}[N / 4 R+(2 K+1)], \\
K=\overline{0,(N / 8 R-1)} \\
S 1_{N / R}(N / 4 R-(2 K+1))=S 1_{N / R}(N / 4 R+(2 K+1)), \\
K=\overline{0,(N / 8 R-1)},
\end{gathered}
$$

тогда с учетом (15)-(18) можно записать $X C(N / 4-(2 K+1))=C 1_{N}(N / 4-(2 K+1))+X C_{N / 2}(N / 4-(2 K+1))$, $X C(N / 4+(2 K+1))=-C 1_{N}(N / 4-(2 K+1))+X C_{N / 2}(N / 4+(2 K+1))$, $X S(N / 4-(2 K+1))=S 1_{N}(N / 4-(2 K+1))+X S_{N / 2}(N / 4-(2 K+1))$, $X S(N / 4+(2 K+1))=S 1_{N}(N / 4-(2 K+1))+X S_{N / 2}(N / 4+(2 K+1))$. 
Пооскольку

$$
\begin{aligned}
& X C_{N / 2}(N / 4-(2 K+1))=X C_{N / 2}(N / 4+(2 K+1)), \\
& X S_{N / 2}(N / 4-(2 K+1))=-X S_{N / 2}(N / 4+(2 K+1)),
\end{aligned}
$$

по (19) - (22) можно выделить

$C 1_{N}(N / 4-(2 K+1))$ и $S 1_{N}(N / 4-(2 K+1))$ :

$C 1_{N}(N / 4-(2 K+1))=(X C(N / 4-(2 K+1))-X C(N / 4+(2 K+1))) / 2$,

$X C_{N / 2}(N / 4-(2 K+1))=(X C(N / 4-(2 K+1))+X C(N / 4+(2 K+1))) / 2$,

$S 1_{N}(N / 4-(2 K+1))=(X S(N / 4-(2 K+1))+X S(N / 4+(2 K+1))) / 2$,

$X S_{N / 2}(N / 4-(2 K+1))=(X S(N / 4-(2 K+1))-X S(N / 4+(2 K+1))) / 2$,

По $X C_{N / 2}$ и $X S_{N / 2}$ вычисляются аналогично $C 1_{N / 2}$ и $S 1_{N / 2}$ и т. д. до $\mathrm{Cl}_{8}(1), \quad S 1_{8}(1), \quad Y 1(0), \quad Y 1(N / 4)$.

Таким образом, обратное нечетное трансформантное преобразование определяется сумма-разностными преобразованиями $(19)-(22)$ и обратными трансформантами.

\section{4. Обратный нечетный трансформант с периодом $N$}

Нечетный трансформант (1) описывается следующими формулами:

$C 1_{N}(G(2 H+1) \pm(2 K+1))=$

$=\sum_{m=0}^{N / 4 G-1}\{\cos (2 \pi(2 H+1)(2 m+1) /(N / G)) R C 1(2 m+1 ; 2 K+1) \mp$

$\mp \sin (2 \pi(2 H+1)(2 m+1) /(N / G)) R S 1(2 m+1 ; 2 K+1)\}=$

$=C 1_{N / G}^{R C 1}(2 H+1 ; 2 K+1) \mp S 1_{N / G}^{R S 1}(2 H+1 ; 2 K+1)$,

$S 1_{N}(G(2 H+1) \pm(2 K+1))=$

$=\sum_{m=0}^{N / 4 G-1}\{\sin (2 \pi(2 H+1)(2 m+1) /(N / G)) R C 1(2 m+1 ; 2 K+1) \pm$

$\pm \cos (2 \pi(2 H+1)(2 m+1) /(N / G) R S 1(2 m+1 ; 2 K+1)=$

$=S 1_{N / G}^{R C 1}(2 H+1 ; 2 K+1) \pm C 1_{N / G}^{R S 1}(2 H+1 ; 2 K+1)$,

$H=\overline{0,(N / 8 G-1)}, \quad K=\overline{0,(G / 4-1)}$,

$C 1_{N}(G \cdot 2 H \pm(2 K+1))=C 1_{N / 2}^{R C 2, R S 2}\left(G H_{ \pm}(2 K+1)\right)$,

$S 1_{N}\left(G \cdot 2 H_{ \pm}(2 K+1)\right)=S 1_{N / 2}^{R C 2, R S 2}(G H \pm(2 K+1))$,

$H=\overline{0, N / 8 G}, \quad K=\overline{0,(G / 4-1)}$.

Из выражений (23), (24) определяем:

$$
\begin{aligned}
& C 1_{N / G}^{R C 1}(2 H+1 ; 2 K+1)= \\
& =\left(C 1_{N}(G(2 H+1)+(2 K+1))+C 1_{N}(G(2 H+1)-(2 K+1))\right) / 2 ; \\
& S 1_{N / G}^{R S 1}(2 H+1 ; 2 K+1)= \\
& =\left(C 1_{N}(G(2 H+1)-(2 K+1))-C 1_{N}(G(2 H+1)+(2 K+1))\right) / 2,
\end{aligned}
$$


$S 1_{N}^{\ddot{R} C 1}(2 \dot{H}+1 ; 2 K+1)=$

$=\left(S 1_{N}(G(2 H+1)+(2 K+1))+S 1_{N}(G(2 H+1)-(2 K+1))\right) / 2$,

$C 1_{N / G}^{R S 1}(2 H+1 ; 2 K+1)=$

$=\left(S 1_{N}(G(2 H+1)+(2 K+1))-S 1_{N}(G(2 H+1)-(2 K+1))\right) / 2$.

Вычисления по формулам (25) и (26) развиваются аналогичным образом после четного-нечетного разбиения $\Pi$.

Теперь по $C_{N / 6}^{R C 1}\left(2 H_{1}+1 ; 2 K+1\right)$,

$S 1_{N / G}^{R S 1}(2 H+1 ; 2 K+1), \quad S 1_{N / G}^{R S 1}(2 H+1 ; 2 K+1)$,

$C 1_{N / G}^{R S 1}(2 H+1 ; 2 K+1)$. следует восстановить

$R C 1(2 m+1 ; 2 K+1)$ и $R S 1(2 m+1 ; 2 K+1)$

как обратные нечетные трансформанты с периодом $N / G$, т. е. вычисления сведены к подобным преобразованиям, причем это нужно продолжать до периодов, поддающихся непосредственному подсчету.

Предположим, что названные процедуры осуществлены: тогда $R C 1(2 m+1 ; 2 K+1)$ и. $R S 1(2 m+1 ; 2 K+1)$ определены, а по $C 1_{N}(G \cdot 2 H \pm(2 K+1))$ и $S 1_{N}(G \cdot 2 H \pm(2 K+1))$ определены также $R\left(22(2 m+1 ; 2 K+1)\right.$ и $R S^{2} 2(2 m+1 ; 2 K+1)$. [ $\left.{ }^{1}\right]$.

На основе формул (49)-(52) $\left\lfloor^{1}\right\rfloor$ находим

$R C(2 m+1 ; 2 K+1)=(R C 1(2 m+1 ; 2 K+1)+R C 2(2 m+1 ; 2 K+1)) / 2$,

$R C(N / 2 G+2 m+1 ; 2 K+1)=$

$=(R C 2(2 m+1 ; 2 K+1)-R C 1(2 m+1 ; 2 K+1)) / 2$,

$R S(2 m+1 ; 2 K+1)=(R S 1(2 m+1 ; 2 K+1)+R S 2(2 m+1 ; 2 K+1)) / 2$,

$R S(N / 2 G+2 m+1 ; 2 K+1)=$

$=(R S 2(2 m+1 ; 2 K+1)-R S 1(2 m+1 ; 2 K+1)) / 2$,

$m=\overline{0,(N / 4 G-1)}$.

Поскольку [ $\left.{ }^{1}\right]$

$$
\begin{aligned}
& R C(Q ; 2 K+1)=\cos (2 \pi Q(2 K+1) / N) X C_{G}^{Q}(2 K+1)- \\
& -\sin (2 \pi Q(2 K+1) / N) X S_{G}^{Q}(2 K+1), \\
& R S(Q ; 2 K+1)=\sin (2 \pi Q(2 K+1) / N) X C_{G}^{Q}(2 K+1)+ \\
& +\cos (2 \pi Q(2 K+1) / N) X S_{G}^{Q}(2 K+1), \\
& Q=2 m+1, \quad m=\overline{0,(N / 2 G-1),}
\end{aligned}
$$

можно определить $X C_{G}^{Q}(2 K+1)$ и $X S \frac{Q}{G}(2 K+1)$ :

$$
\begin{aligned}
X C_{G}^{Q}(2 K+1) & =R C(Q ; 2 K+1) \cos (2 \pi Q(2 K+1) / N)+ \\
& +R S(Q ; 2 K+1) \sin (2 \pi Q(2 K+1) / N), \\
X S_{G}^{Q}(2 K+1) & =R S(Q ; 2 K+1) \cos (2 \pi Q(2 K+1) / N)- \\
& -R C(Q ; 2 K+1) \sin (2 \pi Q(2 K+1) / N) .
\end{aligned}
$$


Учитывая, что

$$
\begin{aligned}
& X C_{G}^{Q}(2 K+1)=\sum_{I=0}^{G / 2-1} Y 1(I N / G+Q) \cos (2 \pi I(2 K+1) / G), \\
& X S_{G}^{Q}(2 K+1)=\sum_{I=0}^{G / 2-1} Y 1(I N / G+Q) \sin (2 \pi I(2 K+1) / G),
\end{aligned}
$$

суть нечетные трансформантные преобразования с периодом $G$, для определения $Y 1(I)$ требуется осуществить обратные преобразования такого же периода для всех $Q$.

Аналогичными преобразованиями определяется $Y 2(I)$ и, в итоге, по формулам (9), (10) - $Y(I)$.

\section{5. Построение алгоритма сокращенного вычисления обратного дискретного преобразования Фурье для заданного объема входного массива $N$}

Полный алгоритм сокращенного вычисления обратного дискретного преобразования Фурье основывается на обратных нечетных трансформантных преобразованиях с периодами $N / R, R=2^{r}, r \in\{0, L P-2\}$ (обозначим как ОТП- $N / R)$, которые после сумма-разностных преобразований (формулы (19)-(22)) сводятся к вычислениям всех обратных нечетных трансформантов с более короткими периодами.

Конструктивно алгоритм ОТП- $N$ начинается с сумма-разностных преобразований, создающих входные потоки данных для обратных нечетных трансформантов, выходные результаты которых четным-нечетным вложением восстанавливают соответствующий исходный массив.

Исходные данные в целом восстанавливаются сумма-разностными преобразованиями (формулы типа (9), (10)).

Если не учитывать операций деления на 2 (в двоичной системе исчисления это означает сдвиг на один разряд) на каждом этапе суммаразностных преобразований (выходные данные можно разделить на $N$ и после выполнения обратного преобразования), то общее количество как операций умножения, так и сложения (вычитания) является таким же, что при прямом преобразовании $\left[{ }^{1}\right]$.

\section{6. Заключение}

Предложенный алгоритм сокращенного вычисления обратного дискретного преобразования Фурье, т. е. алгоритм восстановления вещественного массива по его косинусному и синусному преобразованиям вынуждает принципиально по новому подходить к проблемам, связанным с применением аппарата преобразований тригонометрическими функциями. Если до сих пор считалось естественным рассматривать обратное преобразование Фурье как комплексное, то теперь отпадает необходимость в комплексных преобразованиях вообе. Пара сокращенного прямого $\left[{ }^{1}\right]$ и обратного преобразований вещественного массива обладает существенной эффективностью $\left[{ }^{1}\right]$ по сравнению с известными вычислительными процедурами $\left[{ }^{2}\right]$ и не требует композиционных и декомпозиционных преобразований, присущих комплексным представлениям.

Благодаря малому количеству арифметических операций, алгоритмы сокращенного вычисления прямого и обратного преобразований Фурье вещественного массива обеспечивают сокращенное вычисление также сумм типа сверток $\left[{ }^{2}\right]$.

Предложенная сокращенная процедура вычисления преобразований Фурье может быть использована и при многомерных преобразованиях, например, при обработке изображений $\left[{ }^{3}\right]$. 
1. Арро И. Изв. АН ЭССР. Физ. Матем., 36, № 1, 21-29 (1986).

2. Нуссбаумер $\Gamma$. Быстрое преобразование Фурье и алгоритмы вычисления сверток. М., «Радио и связь», 1985.

3. Быстрые алгоритмы в цифровой обработке изображений. (Под ред. Т. С. Хуанга). М., «Радио и связь», 1984.

Специальное конструкторское бюро вычислительной техники

Поступила в редакцию $11 / \mathrm{V} 1986$

Академии наук Эстонской ССР

I. ARRO

\section{DISKREETSE FOURIER' POORDTEISENDUSE ARVUTAMISE KOONDALGORITM}

Artiklis on vaadeldud kiire Fourier' teisenduse algoritmi arendust, mis seisneb triviaalsete korrutustehete elimineerimises ja originaali esitamises mittekattuvate tsükliliste alamhulkade summana. Toodud algoritm võimaldab sooritada diskreetse Fourier' pöördteisenduse kõige väiksema aritmeetiliste tehete arvuga.

\section{ARRO}

\section{VERKURZTER ALGORITHMUS FUR DIE UMGEKEHRTE DISKRETE FOURIER-TRANSFORMATION}

Es wird die Entwicklung eines Algorithmus zur schnellen umgekehrten FourierTransformation durch Ausschließung der trivialen Multiplikationsoperationen und Vorstellung der Originals als Summe sich nicht deckender zyklischer Mengen behandelt.

Die Zahl aller arithmetischen Operationen für die umgekehrte diskrete FourierTransformation ist bei dem Algorithmus die mindeste. 